Neue Fixkombination

\title{
Dreifach stark gegen Bluthochdruck
}

- Die fixe Dreierkombination eines Sartans mit einem Diuretikum plus einem Kalziumantagonisten erleichert die Compliance der Patienten in der Bluthochdruckbehandlung. Für Hypertoniepatienten, deren Blutdruck mit einer Zweierkombination aus Olmesartan plus Hydrochlorothiazid oder Olmesartan plus Amlodipin und einer zusätzlichen Tablette Amlodipin oder Hydrochlorothiazid auf Zielwert ist, gibt es nun eine Therapieerleichterung: die fixe Dreierkombination Vocado ${ }^{\circledR}$ HCT.

Prof. Rainer Kolloch, Bielefeld, wies darauf hin, dass es nach ESC-Leitlinien in Deutschland derzeit darum gehen müsse, die Zielwerte schneller als bisher zu erreichen, die Medikation insgesamt höher zu dosieren und die Blutdruckwerte in der an
Bluthochdruck erkrankten Bevölkerung weiter herunterzubekommen. Ziel seien Werte unter $140 / 90 \mathrm{mmHg}$. Eine aktuell geführte wissenschaftliche Diskussion um Zielwerte unter 130 bis unter $120 \mathrm{mmHg}$ lege einen falschen Schwerpunkt. „Unser Hauptproblem in der Versorgung ist ein anderes", so Kolloch mit Anspielung auf die Unterversorgung.

\section{Geringeres Nebenwirkungspotenzial} Prof. Günther Linß, Berlin, erläuterte, dass die unterschiedlichen Dosierungen von Vocado ${ }^{\circledast}$ HCT eine Anpassung je nach Wirkung und Verträglichkeit möglich machen, wobei die Kombination in aller Regel weniger Nebenwirkungen mit sich bringe. So ist die Ödemneigung unter Kalziumantago- nisten bei einer Kombination mit einem $\mathrm{AT}_{1}$-Antagonisten geringer als unter einer Kalziumantagonisten-Monotherapie.

Bevorzugte Dosierungen des neuen Präparats sind: $20 \mathrm{mg}$ Olmesartan $/ 5 \mathrm{mg}$ Amlodipin/12,5 mg HCT sowie 40/5/12,5 und 40/10/12,5, erkennbar an der runden Form der Tablette. Die Dosisstärken 40/5/25 und 40/10/25 sind als Tabletten mit ovaler Form verfügbar. Wie Dr. Roger Limberg vom Unternehmen Berlin-Chemie erläuterte, sind die Tabletten im Vergleich zu anderen Präparaten aufgrund der geringen Wirkstoffmenge des Olmesartans sehr klein und können gut geschluckt werden.

- Rainer Klawki

Quelle: Einführungspressekonferenz, Berlin, 26. Januar 2011 (Veranstalter: Berlin-Chemie)

\section{Multidimensionale Lipidtherapie}

\section{Nikotinsäure richtig dosieren, und nicht ohne Statin}

- Mit Statinen gelingt es im Allgemeinen, die Progression der Atherosklerose zu bremsen. Aus Studien, in denen die Wirkung einer lipidsenkenden Therapie auf atherosklerotische Plaques untersucht wurde, folgt, dass die Atherosklerose auch vollständig zum Stillstand gebracht bzw. sogar zurückgebildet werden kann. Dies gelingt ab einem LDL/HDL-Quotienten $<1$.

Nur bei wenigen Patienten kann dieser Quotient allein mit einer Statintherapie erreicht werden. In den meisten Fällen ist dazu ein multidimensionales Lipidmanagement notwendig, welches das atherogene Potenzial sowohl vom LDL-Cholesterin als auch vom HDL-Cholesterin, von Triglyzeriden und $L p(a)$ reduziert. Dies gelingt am besten durch die Kombination eines Statins mit Nikotinsäure.

\section{Wirksamkeit der Nikotinsäure nicht verschenken}

Allerdings muss die Nikotinsäure dazu in der vollen Dosis von zwei Gramm gegeben werden, betonte der Kardiologe und Lipidexperte Prof. John Kastellein, Amsterdam.

\begin{tabular}{|c|c|c|c|}
\hline \multicolumn{4}{|c|}{$\begin{array}{l}\text { Wirksamkeit von } 2 \text { g vs. } 1 \text { g } \\
\text { Nikotinsäure }\end{array}$} \\
\hline & LDL & HDL & Triglyzeride \\
\hline $1 \mathrm{~g} / \mathrm{d}$ & $-9 \%$ & $+15 \%$ & $-11 \%$ \\
\hline $2 \mathrm{~g} / \mathrm{d}$ & $-17 \%$ & $+26 \%$ & $-35 \%$ \\
\hline
\end{tabular}

Wer aus Angst vor Nebenwirkungen Nikotinsäure nur mit einem Gramm dosiert bzw. auf die Dosisverdopplung nach vier Wochen verzichtet, verschenkt einen Großteil der Wirksamkeit (s. Tab. 1).

Die störendste Nebenwirkung der Nikotinsäure ist das Flush-Phänomen. Dieses ist deutlich seltener geworden, seit retardierte Nikotinsäure zusammen mit Laropiprant (als Tredaptive ${ }^{\circledR}$ ) verabreicht wird, welches den Rezeptor in der Haut blockiert, über den der Flush ausgelöst wird. „Der Unterschied zwischen Nikotinsäure und Nikotinsäure plus Laropiprant entspricht etwa einem Flush pro Tag im Vergleich zu einem Flush pro Woche", veranschaulichte Kastellein. Er rät, mit den Pati- enten diese Nebenwirkung zu besprechen und sie zu bitten, diese vor allem in den ersten sechs bis acht Therapiewochen zu ertragen. Nach der Neueinstellung und Dosisverdopplung lässt sie deutlich nach. Auf lange Sicht - nach 20 Wochen - liegt die Flushrate nicht höher als unter Placebo.

\section{Besserer Schutz vor Komplikationen?}

Keineswegs darf das Statin unter einer Therapie mit Nikotinsäure/Laropiprant abgesetzt werden, erklärte Kastellein. Denn Statine sind unverzichtbarer Bestandteil in der Behandlung von Herz-Kreislauf-Erkrankungen. Die langfristige LDL-Cholesterinsenkung verhindert etwa jede dritte kardiovaskuläre Komplikation. Nun hofft man, dass die Kombination Statin plus Nikotinsäure die Komplikationsrate weiter reduziert. Ob dem so ist, wird derzeit in der großen Studie Heart Protection Study 2 Thrive mit über 25000 Hochrisikopatienten untersucht.

\section{- Dr. med. Dirk Einecke}

Quelle: Satellitensymposium, ESC-Jahreskongress 2010, Stockholm (unterstützt von MSD) 